\title{
Models of Supersymmetry for Dark Matter
}

\author{
Carlos Muñoz ${ }^{1,2, a}$ \\ ${ }^{1}$ Departamento de Física Teórica, Universidad Autónoma de Madrid, Cantoblanco, E-28049 Madrid, Spain \\ ${ }^{2}$ Instituto de Física Teórica UAM-CSIC, Campus de Cantoblanco, E-28049 Madrid, Spain
}

\begin{abstract}
A brief review of supersymmetric models and their candidates for dark matter is carried out. The neutralino is a WIMP candidate in the MSSM where $R$-parity is conserved, but this model has the $\mu$ problem. There are natural solutions to this problem that necessarily introduce new structure beyond the MSSM, including new candidates for dark matter. In particular, in an extension of the NMSSM, the right-handed sneutrino can be used for this job. In $R$-parity violating models such as the $\mu \nu \mathrm{SSM}$, the gravitino can be the dark matter, and could be detected by its decay products in gamma-ray experiments.
\end{abstract}

\section{Introduction}

The Higgs particle in the standard model is intriguing, being the only elementary scalar in the spectrum, and introducing the hierarchy problem in the theory. In supersymmetry (SUSY), the presence of the Higgs is more natural: scalar particles exist by construction, the hierarchy problem can be solved, and the models predict that the Higgs mass must be $\lesssim 140 \mathrm{GeV}$ if perturbativity of the relevant couplings up to high-energy scales is imposed. In a sense, the latter has been confirmed by the detection of a scalar particle with a mass of about $125 \mathrm{GeV}$. However, in SUSY at least two Higgs doublets are necessary, and as a consequence new neutral and charged scalars should be detected in the future to confirm the theory. Not only that, as is well known, the spectrum of elementary particles is in fact doubled with masses of about $1 \mathrm{TeV}$, and therefore even the simplest SUSY model, the Minimal Supersymmetric Standard Model (MSSM, see Ref. [1] for a review), predicts a rich phenomenology, including interesting candidates for dark matter (DM) such as the neutralino and sneutrino in $R$-parity conserving $\left(R_{p}\right)$ models and the gravitino in $R$ parity violating $\left(\mathbb{R}_{p}\right)$ models. However, the LHC started operations several years ago and, with Run 1 already finished, SUSY has not been discovered yet. Because of this, it has been raised the question of whether SUSY is still alive. In our opinion the answer is yes, and we think that there are several arguments in favor of it:

- The lower bounds on SUSY particle (sparticle) masses are smaller or about $1 \mathrm{TeV}$, depending on the sparticle analyzed. Thus SUSY masses are still reasonable, and in that sense we can remember that the Higgs particle was discovered with a mass close to its (SUSY) perturbative upper bound.

- Because of the complicated parameter space of SUSY, experimentalists use in their analyses simplified models that do not cover the full MSSM. For example, branching-ratio variations are not considered in much detail, and other assumptions are also made.

a e-mail: c.munoz@uam.es 
- Run 2 is going on, and for the moment with a low luminosity of about $20 \mathrm{fb}^{-1}$. Therefore, to (be prepared) wait for results with higher luminosity seems to be a sensible strategy, since $100 \mathrm{fb}^{-1}$ are expected for the end of Run 2.

- Most searches at the LHC assume $R_{p}$, with the lightest supersymmetric particle (LSP) stable, requiring therefore missing energy in the final state to claim for detection. However, in the case of $\mathbb{R}_{p}$, sparticles can decay to standard model particles, and the bounds on their masses become weaker.

Nevertheless, despite all these arguments in favour of SUSY, it is honest to recognize that it has its own theoretical problems in the low-energy formulation. By construction, the MSSM produces too fast proton decay. In particular, the simultaneous presence of the couplings $\lambda_{i j k}^{\prime} L_{i} Q_{j} d_{k}^{c}$ and $\lambda_{i j k}^{\prime \prime} u_{i}^{c} d_{j}^{c} d_{k}^{c}$ violating lepton $(L)$ and baryon $(B)$ number respectively, as well as $R_{p}$, would produce this effect. The usual assumption in the literature of invoking $R_{p}$ to avoid the problem, forbidding all $R_{p}$ couplings, is perhaps too stringent, since forbidding only one of the above couplings would have been sufficient. We will come back to this point in Section 4. So, once eliminated (all) $B$ and $L$ number violating operators, we are left with the superpotential of the MSSM:

$$
W=Y_{i j}^{e} \hat{H}_{d} \hat{L}_{i} \hat{e}_{j}^{c}+Y_{i j}^{d} \hat{H}_{d} \hat{Q}_{i} \hat{d}_{j}^{c}-Y_{i j}^{u} \hat{H}_{u} \hat{Q}_{i} \hat{u}_{j}^{c}+\mu \hat{H}_{u} \hat{H}_{d},
$$

where $i, j=1,2,3$ are family indexes, and our convention for the contraction of two $S U(2)$ doublets is e.g. $\hat{H}_{u} \hat{H}_{d} \equiv \epsilon_{a b} \hat{H}_{u}^{a} \hat{H}_{d}^{b}$, with $\epsilon_{a b}$ the totally antisymmetric tensor $\epsilon_{12}=1$.

In superpotential (1), the $\mu$ term is necessary e.g. to generate Higgsino masses, given the current experimental lower bound of about $100 \mathrm{GeV}$ on chargino masses. Here we find another problem of SUSY models, the so-called $\mu$ problem [2]. In the presence of a high-energy theory like a grand unified theory (GUT) or a string theory, with a typical scale of the order of $10^{16} \mathrm{GeV}$ or larger, and/or a gravitational theory at the Planck scale, one should be able to explain how to obtain a SUSY mass parameter in the superpotential of the order of the electroweak (EW) scale. The MSSM does not solve the $\mu$ problem. One takes for granted that the $\mu$ term is there, of the order of the EW scale, and that's it. In this sense, the MSSM is a kind of effective theory. Nevertheless, there are natural solutions to this problem that necessarily introduce new structure beyond the MSSM at low energies. Several of these solutions, and the associated SUSY models, will be discussed below.

The work is organized as follows. In Section 2, we will briefly review the popular neutralino DM in the MSSM. We will also see that the left-handed sneutrino is excluded as candidate for DM from experimental constraints. In Section 3, the Next-to-Minimal Supersymmetric Standard Model (NMSSM, see Ref. [3] for a review) is introduced as a solution to the $\mu$ problem, and neutralino DM discussed. In an extension of the NMSSM, we will also see that right-handed sneutrino DM is possible. Finally, in Section 4 we will argue that models with $\mathbb{R}_{p}$ are viable, solving also the $\mu$ problem. In particular, the ' $\mu$ from $\nu$ ' Supersymmetric Standard Model ( $\mu \nu \operatorname{SSM}[4,5]$, see Refs. [6, 7] for reviews), solves the $\mu$-problem through the presence of right-handed neutrino superfields, while simultaneously explains the origin of neutrino masses, i.e. in addition it solves the $v$ problem. Let us emphasize in this sense that in the MSSM, by construction, neutrinos are massless. Of course, the typical sparticle candidates for DM, the neutralino or the right-handed sneutrino, have very short lifetimes in $\mathbb{R}_{p}$ models, and can no longer be used as DM. Nevertheless, the gravitino can be the $\mathrm{DM}$, and we will discuss its feasibility in the $\mu \nu \mathrm{SSM}$, as well as its possible detection in gamma-ray satellite experiments such as the Fermi Large Area Telescope (LAT).

\section{Neutralino DM in the MSSM}

As mentioned in the Introduction, the MSSM superpotential in Eq. (1) conserves by construction $R_{p}$. This is a discrete symmetry which assigns quantum number +1 for particles and -1 for sparticles. 


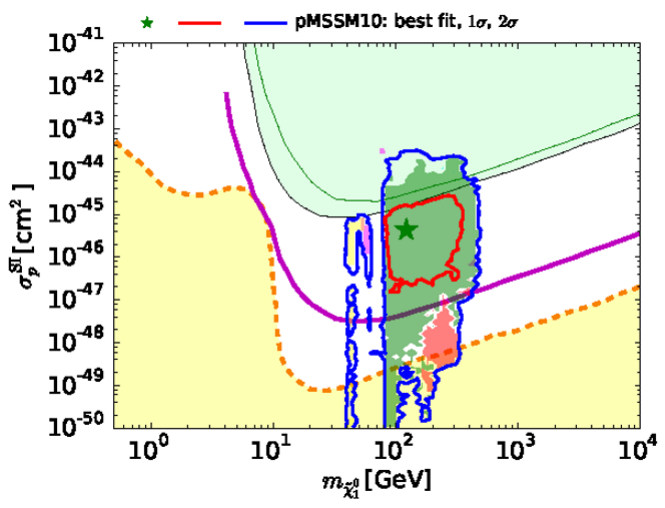

Figure 1. The $\left(m_{\tilde{\chi}_{1}^{0}}, \sigma_{p}^{S I}\right)$ plane in the pMSSM10. The green and black lines show the current sensitivities of the XENON100 and LUX experiments, respectively. The solid purple line show the projected 95\% exclusion sensitivity of the LUX-Zeplin (LZ) experiment, and the dashed orange line show the astrophysical neutrino 'floor', below which astrophysical neutrino backgrounds dominate (yellow region). Figure from [10].

As a consequence, SUSY particles are produced or destroyed only in pairs, and the LSP has to be stable. This implies that the LSP is a possible candidate for DM. In this sense, it is remarkable that in many regions of the parameter space of the MSSM the LSP is the lightest neutralino, a physical superposition of the Bino, and neutral Wino and Higgsinos:

$$
\tilde{\chi}_{1}^{o}=N_{11} \tilde{B}^{0}+N_{12} \tilde{W}^{0}+N_{13} \tilde{H}_{d}^{0}+N_{14} \tilde{H}_{u}^{0} .
$$

Since the neutralino is an electrically neutral particle, it avoids the problem of charged particles as DM: they would bind to nuclei and would be excluded from unsuccessful searches for exotic heavy isotopes (see e.g. Ref. [8] and references therein). Besides, the neutralino is a weakly interacting massive particle (WIMP) and therefore is able to reproduce naturally the amount of relic density that is observed in the Universe, $\Omega_{D M} h^{2} \sim 1$. We can then conclude that, in the MSSM, the lightest neutralino is a very good DM candidate (see Ref. [9] for a review).

The LHC could produce a neutralino with a mass of the order of the GeV-TeV. Such a production and detection would be of course a great success, but not a complete test of the DM theory. Even if we are able to measure the mass and interactions of the new particle, checking whether the amount of relic density is correct, we would never be able to test if the candidate is stable on cosmological scales. A complete confirmation can only arise from experiments where the DM particle is detected as part of the galactic halo or extragalactic structures. This can come from direct and indirect DM searches. Actually, there has been an impressive progress on this issue in recent years, with significant improvements in the precision and sensitivity of experiments. The combination of LHC data with those provided by direct and indirect searches can be a crucial tool for the identification of the DM.

The neutralino WIMP DM could be detected directly in underground laboratories through its elastic interaction with nuclei inside detectors. In view of the LHC1 constraints on SUSY, Higgs data, and flavour physics observables, in Fig. 1 the current constraints on the parameter space of neutralino DM are shown for the phenomenological MSSM, in which 10 of the effective Lagrangian parameters are treated as independent inputs specified at the EW scale (pMSSM10). Indirect DM searches of WIMPs are carried out in neutrino and Cherenkov telescopes, and satellites, through the analysis of the DM annihilation or decay products in the Sun, galactic center, galactic halo or extragalactic 
structures. Such products can be neutrinos, gamma rays and antimatter, and their non-observation put constraints on neutralino DM as well (see Ref. [11] for a review).

\subsection{Sneutrino DM in the MSSM?}

We might wonder whether are there other candidates for DM in the MSSM. In principle, the lefthanded sneutrino fulfils the three interesting properties to become a DM candidate [12, 13]. It is a neutral particle, it is stable when it becomes the LSP, and it is a WIMP. However, at the end of the day, it turns out not to be viable for DM. Given its sizable coupling to the $Z$ boson, left-handed sneutrinos either annihilate too rapidly, resulting in a very small relic abundance, or give rise to a large scattering cross section and are excluded by direct DM searches.

\section{Is there life beyond MSSM/neutralino DM?}

\subsection{Neutralino DM in the NMSSM}

The NMSSM provides an elegant solution to the $\mu$ problem of the MSSM via the introduction of a singlet superfield $\hat{S}$ under the standard model gauge group. Substituting now the $\mu$-term in (1) by

$$
W=\lambda \hat{S} \hat{H}_{u} \hat{H}_{d}+k \hat{S} \hat{S} \hat{S},
$$

when the scalar component of the superfield $\hat{S}$, denoted by $S$, acquires a vacuum expectation value (VEV) of order the SUSY breaking scale, an effective interaction $\mu \hat{H}_{1} \hat{H}_{2}$ is generated through the first term in (3), with $\mu \equiv \lambda\langle S\rangle$. This effective coupling is naturally of order the EW scale if the SUSY breaking scale is not too large compared with $M_{W}$, as expected. In fact, in the NMSSM the EW scale exclusively originates from the SUSY-breaking scale. The second term in (3) is allowed by the gauge symmetry, and avoids, as the $\mu$-term in the MSSM, the existence of a Goldstone boson.

Due to the presence of the superfield $\hat{S}$, in addition to the MSSM fields, the NMSSM contains an extra scalar and pseudoscalar in the Higgs sector, as well as an additional singlino/neutralino. These new fields mix with the corresponding MSSM ones, giving rise to a richer and more complex phenomenology. For example, the results concerning the possible detection of neutralino DM turn out to be modified with respect to those of the MSSM in regions of the parameter space.

\subsection{Sneutrino DM in an extended NMSSM}

An interesting extension of the NMSSM can help to explain the origin of neutrino masses. Since experiments induce us to introduce right-handed neutrino superfields, superpotential (3) can be extended with [14]:

$$
\delta W=Y_{i j}^{v} \hat{H}_{u} \hat{L}_{i} \hat{v}_{j}^{c}+\kappa_{i j} \hat{S} \hat{v}_{i}^{c} \hat{v}_{j}^{c} .
$$

Majorana masses for right-handed neutrinos of the order of the EW scale are generated dynamically through the VEV of the singlet $S, M_{v}=\kappa\langle S\rangle$. This is an example of a seesaw at the EW scale. Light masses are then obtained with a value $m_{v} \simeq Y_{v}^{2} v_{u}^{2} / M_{v}$, which implies Yukawa couplings $Y_{v} \lesssim 10^{-6}$, i.e. of the same order as the electron Yukawa.

As discussed above in the context of the MSSM, the left-handed sneutrino cannot be used as a DM candidate. Actually, a purely right-handed sneutrino either in a natural way, because of its very weak couplings with the rest of the matter implying a scattering cross section too small (supressed by $Y^{v}$ ), and a relic density too large. However, now, through its direct coupling to the singlet in (4), the right-handed sneutrino can be not only a thermal relic DM, but also have a large enough scattering cross section with nuclei as to be detected (see [15] and [16, 17], and references therein). 


\section{Is there life beyond $R_{p} /$ neutralino-sneutrino DM}

As discussed in the Introduction, to impose $R_{p}$ in SUSY models may be too stringent, since the $\mathbb{R}_{p}$ couplings which are harmless for proton decay would also be forbidden. A less drastic solution, taking into account that the choice of $R_{p}$ is ad hoc, is to use other $Z_{N}$ discrete symmetries to forbid only $\lambda_{i j k}^{\prime \prime}$. This is the case e.g. of $Z_{3}$ Baryon-parity [18] which also prohibits dimension-5 proton decay operators, unlike $R_{p}$. In addition, this strategy seems reasonable if one expects all discrete symmetries to arise from the breaking of gauge symmetries of the underlying unified theory, because Baryon-parity and $R_{p}$ are the only two generalized parities which are 'discrete gauge' anomaly free. Actually, this can occur in string compactifications where the matter superfields can have several extra $U(1)$ charges broken spontaneously at high energy, and as a consequence residual $Z_{N}$ symmetries are left in the low-energy theory. The same result can be obtained by the complementary mechanism pointed out in Ref. [5], that stringy selection rules can naturally forbid the $\lambda_{i j k}^{\prime \prime}$ couplings since matter superfields are located in general in different sectors of the compact space.

The gravitino turns out to be an interesting candidate for DM in $\mathbb{R}_{p}$ models. It has an interaction term in the supergravity Lagrangian with the photon and the photino. Since the photino and the neutrinos are mixed in the neutral fermion mass matrix due to the $\mathbb{R}_{p}$, the gravitino will be able to decay into a photon and a neutrino. Nevertheless, this decay is suppressed both by the gravitational interaction (the gravitino is a superWIMP) and by the small $\mathbb{R}_{p}$ coupling, making the gravitino lifetime much longer than the age of the Universe [19]. Adjusting the reheating temperature one can also reproduce the correct relic density.

\subsection{Gravitino DM in the $\mu \nu$ SSM}

Right-handed neutrinos are likely to exist in order to generate neutrino masses. Then, given the fact that sneutrinos are allowed to get VEVs, we may wonder why not to use $\mathbb{R}_{p}$ terms of the type $\hat{v}^{c} \hat{H}_{u} \hat{H}_{d}$ to produce an effective $\mu$ term. This would allow us to solve the $\mu$ problem of the MSSM, without having to introduce an extra singlet superfield as in case of the NMSSM. This is the basic idea of the $\mu \nu \mathrm{SSM}[4,5]$ : natural particle content without the $\mu$ problem. Thus, in addition to the MSSM Yukawa couplings for quarks and charged leptons, the $\mu \nu \mathrm{SSM}$ superpotential contains:

$$
W=-Y_{i j}^{v} \hat{H}_{u} \hat{L}_{i} \hat{v}_{j}^{c}+\lambda_{i} \hat{H}_{u} \hat{H}_{d} \hat{v}_{i}^{c}+\frac{1}{3} \kappa_{i j k} \hat{v}_{i}^{c} \hat{v}_{j}^{c} \hat{v}_{k}^{c}
$$

When the scalar components of the superfields $\hat{v}_{i}^{c}$, denoted by $\tilde{v}_{i R}^{*}$, acquire VEVs of order the EW scale, an effective interaction $\mu \hat{H}_{u} \hat{H}_{d}$ is generated through the second term in (5), with $\mu \equiv \lambda_{i}\left\langle\tilde{v}_{i R}\right\rangle^{*}$. The third term in (5) is allowed by all symmetries, and avoids the presence of a Goldstone boson associated to a global $U(1)$ symmetry, similarly to the case of the NMSSM. In addition, it contributes to generate effective Majorana masses for neutrinos at the EW scale $M_{i j}=\sqrt{2} \kappa_{i j k}\left\langle\tilde{v}_{k R}\right\rangle^{*}$, which together with the Dirac masses generated by the first term, produce correct neutrino masses. Thus, the $\mu \nu \mathrm{SSM}$ solves the $\mu$ and the $v$ problems, by simply introducing right-handed neutrinos.

Since the gravitino decays producing a monochromatic photon with an energy half of the gravitino mass, the prospects for detecting these $\gamma$ rays in satellite experiments can be very interesting, and therefore it seems important to know $\mu \nu \mathrm{SSM}$ predictions concerning gravitino DM detection, first studied in Ref [20]. In recent works [21, 22], a complete analysis of the detection of $\mu \nu \mathrm{SSM}$ gravitino dark matter through $\gamma$-ray observations was carried out. In addition to the two-body decay producing an anisotropic sharp line, the three-body decays producing a smooth spectral signature were included in the analysis. First, a deep exploration of the low-energy parameter space of the $\mu \nu \mathrm{SSM}$ was performed, taking into account that neutrino data must be reproduced. Then, the $\gamma$-ray fluxes predicted 
by the model were compared with Fermi-LAT observations. In particular, with the 95\% CL upper limits on the total diffuse extragalactic $\gamma$-ray background using 50 months of data, together with the upper limits on line emission from an updated analysis using 69.9 months of data. For standard values of bino and wino masses, gravitinos with masses larger than $4 \mathrm{GeV}$, or lifetimes smaller than $10^{28}$ $\mathrm{s}$, produce too large fluxes and are excluded as DM candidates. However, when limiting scenarios with large and close values of the gaugino masses are considered, the constraints turn out to be less stringent, excluding masses larger than $17 \mathrm{GeV}$ and lifetimes smaller than $4 \times 10^{25} \mathrm{~s}$.

\section{Acknowledgments}

This work was supported by grants FPA2015-65929-P MINECO/FEDER UE, Consolider-Ingenio 2010 MultiDark CSD2009-00064, and SEV-2012-0249 'Centro de Excelencia Severo Ochoa'.

\section{References}

[1] S.P. Martin, Adv. Ser. Direct. High Energy Phys. 18 (1998) 1 (1997), hep-ph/9709356

[2] J.E. Kim, H.P. Nilles, Phys. Lett. B138, 150 (1984)

[3] U. Ellwanger, C. Hugonie, A.M. Teixeira, Phys. Rept. 496, 1 (2010), 0910 1785

[4] D.E. López-Fogliani, C. Muñoz, Phys. Rev. Lett. 97, 041801 (2006), hep-ph/0508297

[5] N. Escudero, D.E. López-Fogliani, C. Muñoz, R.R. de Austri, JHEP 12, 099 (2008), 0810 1507

[6] C. Muñoz, in AIP Conf. Proc. (2010), Vol. 1200, p. 413, 0909.5140

[7] C. Muñoz, in $P o S$ (2016), 1608. 07912

[8] A. Kudo, M. Yamaguchi, Phys. Lett. B516, 151 (2001), hep-ph/0103272

[9] C. Muñoz, Int. J. Mod. Phys. A19, 3093 (2004), hep-ph/0309346

[10] E.A. Bagnaschi et al., Eur. Phys. J. C75, 500 (2015), 1508.01173

[11] C. Muñoz, Nucl. Instrum. Meth. A692, 13 (2012), 1203.0678

[12] L.E. Ibañez, Phys. Lett. B137, 160 (1984)

[13] J.S. Hagelin, G.L. Kane, S. Raby, Nucl. Phys. B241, 638 (1984)

[14] R. Kitano, K.y. Oda, Phys. Rev. D61, 113001 (2000), hep-ph/9911327

[15] D.G. Cerdeño, C. Muñoz, O. Seto, Phys. Rev. D79, 023510 (2009), 0807 . 3029

[16] D.G. Cerdeño, M. Peiró, S. Robles, JCAP 1408, 005 (2014), 1404 . 2572

[17] D.G. Cerdeño, M. Peiró, S. Robles, Phys. Rev. D91, 123530 (2015), 1501.01296

[18] L.E. Ibanez, G.G. Ross, Nucl. Phys. B368, 3 (1992)

[19] F. Takayama, M. Yamaguchi, Phys. Lett. B485, 388 (2000), hep-ph/0005214

[20] K.Y. Choi, D.E. López-Fogliani, C. Muñoz, R.R. de Austri, JCAP 03, 028 (2010), 0906. 3681

[21] A. Albert, G. Gomez-Vargas, M. Grefe, C. Muñoz, C. Weniger, E. Bloom, E. Charles, M. Mazziotta, A. Morselli, JCAP 10, 023 (2014), 1406. 3430

[22] G.A. Gomez-Vargas, D.E. López-Fogliani, C. Muñoz, A.D. Pérez, R. Ruiz de Austri (2016), 1608.08640 